\title{
Transport of Relativistic Electrons Scattered by the Coulomb Force and a Thermionic Energy Converter with a Built-in Discharge Tube
}

\author{
Mitsuaki Nagata \\ Soft Creator Company, Kyoto, Japan \\ Email: nagata@heian-kogyo.jp
}

How to cite this paper: Nagata, M. (2021) Transport of Relativistic Electrons Scattered by the Coulomb Force and a Thermionic Energy Converter with a Built-in Discharge Tube. Journal of Modern Physics, 12, 17081720.

https://doi.org/10.4236/jmp.2021.1212099

Received: September 16, 2021

Accepted: October 24, 2021

Published: October 27, 2021

Copyright $\odot 2021$ by author(s) and Scientific Research Publishing Inc. This work is licensed under the Creative Commons Attribution International License (CC BY 4.0).

http://creativecommons.org/licenses/by/4.0/

\begin{abstract}
A transport equation of momentum for relativistic electrons scattered isotropically was previously reported. Here, a momentum-transport equation for relativistic electrons "scattered anisotropically" by the Coulomb force is inquired into. An ideal plasma consisting of electrons and deuterons is treated again. Also, to raise a generation-ability of a thermionic energy converter, a means of introducing external electric and magnetic fields within "a converter in which an emitter plate and a collector plate face simply each other" is proposed.
\end{abstract}

\section{Keywords}

Transport of Relativistic Electrons, Coulomb Force Scattering, Thermionic Energy Converter with Some Supplemental Equipments

\section{Introduction}

In the classical theory (based on the Boltzmann equation with the Fokker-Planck collision term) with respect to the electron transport in an ideal plasma consisting of electrons and deuterons, as a frictional force to suppress unlimited increase of a drift velocity by an external electric field, only the dynamical frictional force coming from the cumulative effect of small angle deflections ceaselessly occurring is generally taken into consideration. However, considering that rare large angle deflections ought to be the scatterings due to the two-body (an electron and a deuteron) collisions, we reported [1] about the evaluation of an effective radius of the Coulomb force of a deuteron. The unexpected result was that a frictional force coming from the two-body collisions is much stronger than the dynamical frictional one (from Equations (18)-(20) of Ref. [1]). So, for simplifi- 
cation of an analysis in this research, we disregard an effect of the many-body collisions on a drift movement of an electron, compared with one of the two-body collisions. Furthermore, assuming that every electron has a mean thermal velocity $\bar{v}$, we inquire into a transport equation of momentum for relativistic electrons in the ideal plasma.

For changing radiation energy from a huge-sided magnetic mirror reactor into electric energy in future, a thermionic energy converter [2]-[9] is considered to be a promising generator, next to a steam turbine one. We discuss in Section 4 about a way to raise a generation-efficiency of a converter by help of some supplemental equipments.

\section{Momentum Transport Equation}

This research is discussed under the presupposition that a deuteron has as effective radius $P_{\mathrm{up}-\mathrm{r}}$ of the Coulomb force, with respect to the two-body collisions. Then, a mean collision frequency $v_{\mathrm{r}}$ of an electron is $n_{\mathrm{p}} \pi p_{\mathrm{up}-\mathrm{r}}^{2} \bar{v}\left(\equiv \bar{v} / \lambda_{\mathrm{r}}, n_{\mathrm{p}}\right.$ is a deuteron density which is equal to an electron density $n_{\mathrm{e}}$ ). A value of $P_{\text {up-r }}$ is estimated in after (24), together with $\varsigma_{\mathrm{r}}$ appearing later.

We first consider the case where a small electric field $\mathbf{E}(t)$ and a magnetic field B are:

$$
\left\{\begin{array}{l}
\mathbf{E}(t)=-\hat{z} E \cos \omega t \\
\mathbf{B}=\hat{y} B
\end{array} \quad(t \text { is time, } \omega \text { is a frequency) }\right.
$$

We use four coordinate systems:

$$
\left\{\begin{array}{l}
\text { the orthogonal coordinates }(x, y, z),\left(x^{\prime}, y^{\prime}, z^{\prime}\right) \\
\text { the polar coorinates }(\theta, \phi, z),\left(\theta^{\prime}, \phi^{\prime}, z^{\prime}\right)
\end{array}\right.
$$

Here, $y / / y^{\prime}$ and the $z^{\prime}$-axis is in the direction of an electron drift velocity $\mathbf{u}(t)$ which is both on the $x-z$ plane and on the $x^{\prime}-z^{\prime}$ plane. The angles $\theta$ and $\theta^{\prime}$ are angles between a velocity variable $v$ and the $z$-axis and between $v$ and the $z^{\prime}$-axis, respectively. The angle $\phi$ and $\phi^{\prime}$ are inclinations from the $x$-axis, the $x^{\prime}$-axis on the $x$ - $y$ plane, respectively. We assume that both a temperature distribution and a density distribution, with respect to electrons, are uniform in space and that $|\mathbf{u}(t)| \ll \bar{v}<0.1 c \quad$ (c. the light speed).

The linearized relativistic equation of motion for an electron having a velocity $v\left(t, t_{0}\right)$ at time $t$ after having been scattered with a velocity $v\left(t_{0}\right)$ at past time $t_{0}$ by a deuteron is given by (2) of Ref. [10]

$$
\frac{m_{\mathrm{e}}}{\left(1-\frac{v\left(t_{0}\right)^{2}}{c^{2}}\right)^{1 / 2}} \frac{\partial \boldsymbol{v}\left(t, t_{0}\right)}{\partial t}=-\frac{\alpha^{\prime \prime}}{c^{2}} \frac{\partial \boldsymbol{v}_{\mathrm{t}}}{\partial t}-\frac{\boldsymbol{v}_{\mathrm{t}}}{c^{2}}\left(-q \mathbf{E}(t) \cdot \boldsymbol{v}_{\mathrm{t}}\right)-q \mathbf{E}(t)-q v\left(t, t_{0}\right) \times \mathbf{B}
$$

Here, $m_{\mathrm{e}}$ is the rest mass of an electron, $-q$ is an electron charge,

$v_{\mathrm{t}}=\boldsymbol{v}\left(t, t_{0}\right)_{(\mathrm{E}(t)=0)}, \quad \alpha^{\prime \prime}=\int_{t_{0}}^{t}-q \mathrm{E}(t) \cdot v_{\mathrm{t}} \mathrm{d} t$. We note that (2) can be regarded to be the equation of motion for an electron with a constant mass 
$m_{\mathrm{e}} /\left(1-\boldsymbol{v}\left(t_{0}\right)^{2} / c^{2}\right)^{1 / 2}$

A momentum transport-equation is written as

$$
\begin{aligned}
& \sum\left\{\frac{m_{\mathrm{e}}}{\left(1-\frac{\boldsymbol{v}\left(t_{0}\right)^{2}}{c^{2}}\right)^{1 / 2}} \frac{\partial \boldsymbol{v}\left(t, t_{0}\right)}{\partial t}+\frac{\alpha^{\prime \prime}}{c^{2}} \frac{\partial \boldsymbol{v}_{\mathrm{t}}}{\partial t}+\frac{\boldsymbol{v}_{\mathrm{t}}}{c^{2}}\left(-q \mathbf{E}(t) \cdot \boldsymbol{v}_{\mathrm{t}}\right)\right\} \\
& =\sum\left\{-q \mathbf{E}(t)-q \boldsymbol{v}\left(t, t_{0}\right) \times \mathbf{B}\right\}+\boldsymbol{P}_{\text {after }}-\boldsymbol{P}_{\text {before }}
\end{aligned}
$$

Here, $\sum$ represents summation of the vector quantity of each term over electrons per unit volume. And with respect to electrons scattered by collisions with deuterons per unit volume and per unit time at time $t, \boldsymbol{P}_{\text {after }}$ and $\boldsymbol{P}_{\text {before }}$ are total momentum of those electrons just after the collisions and just before the collisions, respectively.

1) About $\boldsymbol{P}_{\text {after }}$ in (3)

The number of electrons scattered by deuterons is $n_{\mathrm{e}} v_{\mathrm{r}}$ per unit volume and per unit time. For a velocity distribution of those $n_{\mathrm{e}} v_{\mathrm{r}}$ electrons just after the collisions, we assume again such a spherical surface as shown in Figure 1 of Ref. [11]:

$$
n_{\mathrm{e}} v_{\mathrm{r}}\left[\left(\frac{\bar{v}+2 \zeta_{\mathrm{r}} u(t) \cos \theta^{\prime}}{\bar{v}}\right) \frac{\sin \theta \mathrm{d} \phi \mathrm{d} \theta}{4 \pi}\right] \delta(v-v(t)) \mathrm{d} v=\mathrm{d} \Phi(t)
$$

The quantity in the above bracket is the solid angle element in the direction of $(\theta, \phi), v$ is the magnitude of a velocity variable $v$ of an electron, $u(t)=|\mathbf{u}(t)|, v(t)=\bar{v}+\zeta_{\mathrm{r}} u(t) \cos \theta^{\prime} \quad\left(\zeta_{\mathrm{r}}\right.$ is a remaining ratio of $\mathbf{u}(t)$ in the relativistic case) and $\delta(\cdots)$ is a delta-function. $\boldsymbol{P}_{\text {after }}$ is given by

$$
\begin{aligned}
P_{\text {after }}= & \int_{\theta^{\prime}=0}^{\pi} \int_{\phi^{\prime}=0}^{2 \pi} \int_{v=0}^{\infty} n_{\mathrm{e}} v_{\mathrm{r}}\left[\left(\frac{\bar{v}+2 \zeta_{\mathrm{r}} u(t) \cos \theta^{\prime}}{\bar{v}}\right) \frac{\sin \theta^{\prime} \mathrm{d} \phi^{\prime} \mathrm{d} \theta^{\prime}}{4 \pi}\right] \\
& \times \delta(v-v(t)) \mathrm{d} v \frac{m_{\mathrm{e}} v^{\prime}}{\left(1-\frac{\left(v^{\prime}\right)^{2}}{c^{2}}\right)^{1 / 2}}
\end{aligned}
$$

Here, $v^{\prime}=\hat{x}^{\prime} v \sin \theta^{\prime} \cos \phi^{\prime}+\hat{y}^{\prime} v \sin \theta^{\prime} \sin \phi^{\prime}+\hat{z}^{\prime} v \cos \theta^{\prime}$. Using the following approximation:

$$
\begin{aligned}
& \frac{m_{\mathrm{e}}}{\left(1-\frac{\left(\bar{v}+\zeta_{\mathrm{r}} u(t) \cos \theta^{\prime}\right)^{2}}{c^{2}}\right)^{1 / 2}} \simeq \frac{m_{\mathrm{e}}}{\left[\left(1-\frac{\bar{v}^{2}}{c^{2}}\right)\left(1-\frac{2 \bar{v} \zeta_{\mathrm{r}} u(t) \cos \theta^{\prime}}{c^{2}}\right)\right]^{1 / 2}} \\
& \simeq \frac{m_{\mathrm{e}}}{\gamma_{\mathrm{r}}}\left(1+\frac{\bar{v} \zeta_{\mathrm{r}} u(t) \cos \theta^{\prime}}{c^{2}}\right)\left(\text { where, } \gamma_{\mathrm{r}}=\left(1-\bar{v}^{2} / c^{2}\right)^{1 / 2}\right),
\end{aligned}
$$

we have

$$
\boldsymbol{P}_{\mathrm{after}} \simeq n_{\mathrm{e}} v_{\mathrm{r}} \frac{m_{\mathrm{e}}}{\gamma_{\mathrm{r}}} \hat{z}^{\prime} \zeta_{\mathrm{r}} \mathbf{u}(t)\left(1+\frac{\bar{v}^{2}}{3 c^{2}}\right)=n_{\mathrm{e}} v_{\mathrm{r}} \frac{m_{\mathrm{e}}}{\gamma_{\mathrm{r}}} \zeta_{\mathrm{r}} \mathbf{u}(t)\left(1+\frac{\bar{v}^{2}}{3 c^{2}}\right)
$$


The term with products or squares of the drift velocity and the electric field have been neglected in (6) and (7). We will do so also in later calculations.

2) About $\boldsymbol{P}_{\text {before }}$ in (3)

The number of electrons scattered with velocity magnitudes $v \sim v+\mathrm{d} v$ in the direction of $(\theta, \phi)$ during the time interval $t_{0} \sim t_{0}+\mathrm{d} t_{0}$ before time $t$ is $\mathrm{d} \Phi\left(t_{0}\right) \mathrm{d} t_{0}$ per unit volume. Of these electrons, the number of electrons having not collided until time $t$ is $\mathrm{d} \Phi\left(t_{0}\right) \mathrm{d} t_{0} \exp \left[-\int_{t_{0}}^{t} \frac{v\left(t, t_{0}\right)_{\left(v=v\left(t_{0}\right)\right)}}{\lambda_{\mathrm{r}}} \mathrm{d} t\right]$, where $v\left(t, t_{0}\right)$ is the magnitude of a velocity $v\left(t, t_{0}\right)$ at time $t$ after having been scattered with a velocity $v\left(t_{0}\right)=\bar{v}+\zeta_{\mathrm{r}} u\left(t_{0}\right) \cos \theta^{\prime}$. The velocity $v\left(t, t_{0}\right)$ is given after by (9). These electrons have, at time $t$, momentum

$$
\frac{m_{\mathrm{e}}}{\left(1-\frac{v\left(t_{0}\right)^{2}}{c^{2}}\right)^{1 / 2}} \boldsymbol{v}\left(t, t_{0}\right)_{\left(v=\nu\left(t_{0}\right)\right)},
$$

and probability by which these electrons are scattered per unit time at time $t$ is $v\left(t, t_{0}\right)_{\left(v=v\left(t_{0}\right)\right)} / \lambda_{\mathrm{r}}$. Accordingly, $\boldsymbol{P}_{\text {before }}$ is given by

$$
\begin{aligned}
\boldsymbol{P}_{\text {before }}= & \int_{t_{0}=-\infty}^{t} \int_{\theta=0}^{\pi} \int_{\phi=0}^{2 \pi} n_{\mathrm{e}} v_{\mathrm{r}} \mathrm{d} t_{0}\left[\frac{\bar{v}+2 \zeta_{\mathrm{r}} \mathbf{u}(t) \cos \theta^{\prime}}{\bar{v}} \times \frac{\sin \theta \mathrm{d} \phi \mathrm{d} \theta}{4 \pi}\right] \\
& \times \exp \left[-\int_{t_{0}}^{t} \frac{\boldsymbol{v}\left(t, t_{0}\right)_{\left(v=\nu\left(t_{0}\right)\right)}}{\lambda_{\mathrm{r}}} \mathrm{d} t\right] \frac{\boldsymbol{v}\left(t, t_{0}\right)_{\left(v=\nu\left(t_{0}\right)\right)}}{\lambda_{\mathrm{r}}} \\
& \times \frac{m_{\mathrm{e}}}{\left(1-\frac{v\left(t_{0}\right)^{2}}{c^{2}}\right)^{1 / 2}} \boldsymbol{v}\left(t, t_{0}\right)_{\left(\nu=\nu\left(t_{0}\right)\right)}
\end{aligned}
$$

We substitute the following three relationships into (8).

a) Equation (6) $\left(t \rightarrow t_{0}\right)$

b) Equation (9) below:

From the energy relationship

$\frac{m_{\mathrm{e}} c^{2}}{\left(1-\frac{v\left(t, t_{0}\right)_{\left(v=\nu\left(t_{0}\right)\right)}^{2}}{c^{2}}\right)^{1 / 2}}-\frac{m_{\mathrm{e}} c^{2}}{\left(1-\frac{v\left(t_{0}\right)^{2}}{c^{2}}\right)^{1 / 2}}=\int_{t_{0}}^{t}-q \mathbf{E}(t) \cdot\left(v\left(t, t_{0}\right)_{(\mathbf{E}(t)=0, v=\bar{v})}\right) \mathrm{d} t \equiv \alpha_{(v=\bar{v})}^{\prime \prime}$,

we have

$$
v\left(t, t_{0}\right)_{\left(v=v_{0}(t)\right)} \simeq v\left(t_{0}\right)+\frac{\gamma_{\mathrm{r}}^{3}}{m_{\mathrm{e}} \overline{\bar{v}}} \alpha_{(v=\bar{\nu})}^{\prime \prime} \quad\left(\left|\alpha_{(v=\bar{\nu})}^{\prime \prime}\right| \ll m_{\mathrm{e}} c^{2} / \gamma_{\mathrm{r}}\right)
$$

where, with $\omega_{\text {cr }}=q|\mathbf{B}| \gamma_{\mathrm{r}} / m_{\mathrm{e}}$ and

$$
\begin{aligned}
& \boldsymbol{v}\left(t, t_{0}\right)_{(E(t)=0, v=\bar{v})}=\boldsymbol{v}_{t_{(v=\bar{v})}} \\
& =\hat{x}\left[\bar{v} \sin \theta \cos \phi \cos \omega_{\mathrm{cr}}\left(t-t_{0}\right)+\bar{v} \cos \theta \sin \omega_{\mathrm{cr}}\left(t-t_{0}\right)\right]+\hat{y}[\bar{v} \sin \theta \sin \phi] \\
& +\hat{z}\left[\bar{v} \cos \theta \cos \omega_{\mathrm{cr}}\left(t-t_{0}\right)-\bar{v} \sin \theta \cos \phi \sin \omega_{\mathrm{cr}}\left(t-t_{0}\right)\right],
\end{aligned}
$$




$$
\begin{aligned}
\alpha_{(v=\bar{v})}^{\prime \prime}= & \frac{q \mathbf{E}}{\omega_{\mathrm{cr}}^{2}-\omega^{2}}\left\{\bar{v} \sin \theta \cos \phi\left[\omega_{\mathrm{cr}} \cos \omega t \cos \omega_{\mathrm{cr}}\left(t-t_{0}\right)+\omega \sin \omega t \sin \omega_{c r}\left(t-t_{0}\right)\right]\right. \\
& +\bar{v} \cos \theta\left[\omega_{\mathrm{cr}} \cos \omega t \sin \omega_{\mathrm{cr}}\left(t-t_{0}\right)-\omega \sin \omega t \cos \omega_{\mathrm{cr}}\left(t-t_{0}\right)\right] \\
& \left.-\bar{v} \sin \theta \cos \phi\left(\omega_{\mathrm{cr}} \cos \omega t_{0}\right)+\bar{v} \cos \theta\left(\omega \sin \omega t_{0}\right)\right\}
\end{aligned}
$$

c) An Equation (11) below, for a drift velocity of electrons,

$$
\begin{aligned}
n_{\mathrm{e}} \mathbf{u}(t)= & \int_{t_{0}} \int_{\theta} \int_{\phi} n_{\mathrm{e}} v_{\mathrm{r}} \mathrm{d} t_{0}\left(1+\frac{2 \zeta_{\mathrm{r}} \mathbf{u}\left(t_{0}\right) \cos \theta^{\prime}}{\bar{v}}\right) \frac{\sin \theta \mathrm{d} \phi \mathrm{d} \theta}{4 \pi} \\
& \times \exp \left[-\int_{t_{0}}^{t} \frac{\left.v\left(t, t_{0}\right)_{\left(v=v\left(t_{0}\right)\right)} \mathrm{d} t\right] \boldsymbol{v}\left(t, t_{0}\right)_{\left(v=v\left(t_{0}\right)\right)}}{\lambda_{\mathrm{r}}}\right.
\end{aligned}
$$

Then, (8) becomes

$$
\begin{aligned}
\boldsymbol{P}_{\text {before }}= & n_{\mathrm{e}} \frac{m_{\mathrm{e}}}{\gamma_{\mathrm{r}}} v_{\mathrm{r}} \mathbf{u}(t)+\int_{t_{0}, \theta, \phi} n_{\mathrm{e}} v_{\mathrm{r}} \mathrm{d} t_{0} \frac{\sin \theta \mathrm{d} \phi \mathrm{d} \theta}{4 \pi} \exp \left[-v_{\mathrm{r}}\left(t-t_{0}\right)\right] \frac{m_{\mathrm{e}}}{\gamma_{\mathrm{r}} \lambda_{\mathrm{r}}}\left(1+\frac{\bar{v}^{2}}{c^{2}}\right) \\
& \times \varsigma_{\mathrm{r}} u\left(t_{0}\right) \cos \theta^{\prime} \boldsymbol{v}\left(t, t_{0}\right)_{(E(t)=0, v=\bar{\nu})}+\int_{t_{0}, \theta, \phi} n_{\mathrm{e}} v_{\mathrm{r}} \mathrm{d} t_{0} \frac{\sin \theta \mathrm{d} \phi \mathrm{d} \theta}{4 \pi} \\
& \times \exp \left[-v_{\mathrm{r}}\left(t-t_{0}\right)\right] \frac{m_{\mathrm{e}}}{\gamma_{\mathrm{r}} \lambda_{\mathrm{r}}} \cdot \frac{\gamma_{\mathrm{r}}^{3}}{m_{\mathrm{e}} \bar{\nu}} \alpha_{(v=\bar{\nu})}^{\prime \prime} \boldsymbol{v}\left(t, t_{0}\right)_{(\mathrm{E}(t)=0, v=\bar{v})}
\end{aligned}
$$

Furthermore, in order to calculate the second term in the right-hand side (RHS) of (12), we express $\mathbf{u}\left(t_{0}\right)$ by the following form:

$$
\mathbf{u}\left(t_{0}\right)=\hat{x} u_{x}\left(t_{0}\right)+\hat{z} u_{z}\left(t_{0}\right)
$$

And we use the following relationships

$$
\left\{\begin{array}{l}
\left|\mathbf{u}\left(t_{0}\right)\right| \cos \theta^{\prime}=u\left(t_{0}\right) \cos \theta^{\prime}=u_{x}\left(t_{0}\right) \sin \theta \cos \phi+u_{z}\left(t_{0}\right) \cos \theta \\
u_{x}\left(t_{0}\right)=\operatorname{Re}\left[\left(u_{x R}+i u_{x I}\right) \mathrm{e}^{\mathrm{i} \omega t_{0}}\right]=u_{x R} \cos \omega t_{0}-u_{x I} \sin \omega t_{0} \\
u_{z}\left(t_{0}\right)=\operatorname{Re}\left[\left(u_{z R}+i u_{z I}\right) \mathrm{e}^{\mathrm{i} \omega t_{0}}\right]=u_{z R} \cos \omega t_{0}-u_{z I} \sin \omega t_{0}
\end{array}\right.
$$

Then, the 2nd term in RHS of (12)

$$
\begin{aligned}
= & n_{\mathrm{e}} v_{\mathrm{r}}^{2} \frac{m_{\mathrm{e}}}{\gamma_{\mathrm{r}}} \varsigma_{\mathrm{r}}\left(1+\frac{\bar{v}^{2}}{c^{2}}\right) \frac{1}{3} \int_{t_{0}=-\infty}^{t} \mathrm{~d} t_{0} \exp \left[-v_{\mathrm{r}}\left(t-t_{0}\right)\right]\left[\hat{x}\left(u_{x R} \cos \omega t_{0}-u_{x I} \sin \omega t_{0}\right)\right. \\
& \times \cos \left[\omega_{\mathrm{cr}}\left(t-t_{0}\right)\right]-\hat{z}\left(u_{x R} \cos \omega t_{0}-u_{x I} \sin \omega t_{0}\right) \sin \left[\omega_{\mathrm{cr}}\left(t-t_{0}\right)\right]+\hat{x}\left(u_{z R} \cos \omega t_{0}\right. \\
& \left.\left.-u_{z I} \sin \omega t_{0}\right) \sin \left[\omega_{\mathrm{cr}}\left(t-t_{0}\right)\right]+\hat{z}\left(u_{z R} \cos \omega t_{0}-u_{z I} \sin \omega t_{0}\right) \cos \left[\omega_{\mathrm{cr}}\left(t-t_{0}\right)\right]\right] \\
= & n_{\mathrm{e}} v_{\mathrm{r}} \frac{m_{\mathrm{e}}}{\gamma_{\mathrm{r}}} \varsigma_{\mathrm{r}}\left(1+\frac{\bar{v}^{2}}{c^{2}}\right)\left[\hat{x}\left(\beta_{2}+\frac{\beta_{2}^{\prime}}{\omega} \frac{\partial}{\partial t}\right) u_{x}(t)+\hat{z}\left(\beta_{1}+\frac{\beta_{1}^{\prime}}{\omega} \frac{\partial}{\partial t}\right) u_{x}(t)\right. \\
& \left.+\hat{x}\left(\beta_{1}+\frac{\beta_{1}^{\prime}}{\omega} \frac{\partial}{\partial t}\right)\left(-u_{z}(t)\right)+\hat{z}\left(\beta_{2}+\frac{\beta_{2}^{\prime}}{\omega} \frac{\partial}{\partial t}\right) u_{z}(t)\right]
\end{aligned}
$$

where, $u_{x}(t)=u_{x}\left(t_{0}\right)_{\left(t_{0} \rightarrow t\right)}, u_{z}(t)=u_{z}\left(t_{0}\right)_{\left(t_{0} \rightarrow t\right)}$, and $\left(\beta_{1}, \beta_{1}^{\prime}, \beta_{2}, \beta_{2}^{\prime}\right)$ together with $\left(\beta_{20}, \beta_{20}^{\prime}\right)$ are shown in after (21). Equation (13) can be generalized as:

The 2nd term in RHS of (12) 


$$
\begin{aligned}
= & n_{\mathrm{e}} v_{r} \frac{m_{\mathrm{e}}}{\gamma_{\mathrm{r}}} \varsigma_{\mathrm{r}}\left(1+\frac{\bar{v}^{2}}{c^{2}}\right) \times\left[\left(\beta_{1}+\frac{\beta_{1}^{\prime}}{\omega} \frac{\partial}{\partial t}\right)(\mathbf{u}(t) \times \hat{b})+\left(\beta_{2}+\frac{\beta_{2}^{\prime}}{\omega} \frac{\partial}{\partial t}\right) \hat{b} \times(\mathbf{u}(t) \times \hat{b})\right. \\
& \left.+\left(\beta_{20}+\frac{\beta_{20}^{\prime}}{\omega} \frac{\partial}{\partial t}\right) \hat{b}(\mathbf{u}(t) \cdot \hat{b})\right]
\end{aligned}
$$

Next, the 3rd term in RHS of (12) becomes

$n_{\mathrm{e}} \gamma_{\mathrm{r}}^{2} q E\left[\hat{x}\left(-\beta_{1} \cos \omega t+\beta_{1}^{\prime} \sin \omega t\right)+\hat{z}\left(\beta_{2} \cos \omega t-\beta_{2}^{\prime} \sin \omega t\right)\right]$. This can be generalized as:

The 3rd term in RHS of (12)

$$
\begin{aligned}
= & n_{\mathrm{e}} \gamma_{\mathrm{r}}^{2}\left[\left(\beta_{1}+\frac{\beta_{1}^{\prime}}{\omega} \frac{\partial}{\partial t}\right)(-q \mathbf{E}(t) \times \hat{b})+\left(\beta_{2}+\frac{\beta_{2}^{\prime}}{\omega} \frac{\partial}{\partial t}\right) \hat{b} \times(-q \mathbf{E}(t) \times \hat{b})\right. \\
& \left.+\left(\beta_{20}+\frac{\beta_{20}^{\prime}}{\omega} \frac{\partial}{\partial t}\right) \hat{b}(-q \mathbf{E}(t) \cdot \hat{b})\right]
\end{aligned}
$$

Accordingly, the momentum transfer term in the field of the two-body (electron-deuteron) collisions is given by

$$
\begin{aligned}
\boldsymbol{P}_{\text {after }}-\boldsymbol{P}_{\text {before }}= & -n_{\mathrm{e}} v_{r} \frac{m_{\mathrm{e}}}{\gamma_{\mathrm{r}}} \mathbf{u}(t)\left[1-\varsigma_{r}\left(1+\frac{\bar{v}^{2}}{3 c^{2}}\right)\right]-n_{\mathrm{e}}\left[\left(\beta_{1}+\frac{\beta_{1}^{\prime}}{\omega} \frac{\partial}{\partial t}\right)\left(\boldsymbol{F}_{\mathrm{c}} \times \hat{b}\right)\right. \\
& \left.+\left(\beta_{20}+\frac{\beta_{20}^{\prime}}{\omega} \frac{\partial}{\partial t}\right) \hat{b}\left(\boldsymbol{F}_{\mathrm{c}} \cdot \hat{b}\right)+\left(\beta_{2}+\frac{\beta_{2}^{\prime}}{\omega} \frac{\partial}{\partial t}\right) \hat{b} \times\left(\boldsymbol{F}_{\mathrm{c}} \times \hat{b}\right)\right]
\end{aligned}
$$

where,

$$
\boldsymbol{F}_{\mathrm{c}}=-q \mathbf{E}(t) \gamma_{\mathrm{r}}^{2}+v_{r} \frac{m_{\mathrm{e}}}{\gamma_{\mathrm{r}}} \varsigma_{r}\left(1+\frac{\bar{v}^{2}}{c^{2}}\right) \mathbf{u}(t)
$$

3) About $\sum \frac{\alpha^{\prime \prime}}{c^{2}} \frac{\partial v_{t}}{\partial t}$ in (3)

$$
\begin{aligned}
& \sum \frac{\alpha^{\prime \prime}}{c^{2}} \frac{\partial v_{t}}{\partial t}=\int_{t_{0}} \int_{\theta} \int_{\phi} \frac{\alpha_{(v=\bar{v})}^{\prime \prime}}{c^{2}} \frac{\partial \boldsymbol{v}_{t_{(v=\bar{v})}}}{\partial t} n_{\mathrm{e}} v_{r} \mathrm{~d} t_{0} \frac{\sin \theta \mathrm{d} \phi \mathrm{d} \theta}{4 \pi} \exp \left[-\frac{\bar{v}}{\lambda_{r}}\left(t-t_{0}\right)\right] \\
& =n_{\mathrm{e}} \frac{\omega_{\mathrm{cr}}}{v_{r}} \frac{\bar{v}^{2}}{c^{2}}\left\{-\left(\beta_{2}+\frac{\beta_{2}^{\prime}}{\omega} \frac{\partial}{\partial t}\right)(-q \mathbf{E}(t) \times \hat{b})+\left(\beta_{1}+\frac{\beta_{1}^{\prime}}{\omega} \frac{\partial}{\partial t}\right) \hat{b} \times(-q \mathbf{E}(t) \times \hat{b})\right\}
\end{aligned}
$$

4) About $\sum \frac{\boldsymbol{v}_{t}}{c^{2}}\left[-q \mathbf{E}(t) \cdot \boldsymbol{v}_{t}\right]$ in (3)

$$
\begin{aligned}
& \sum \frac{v_{t}}{c^{2}}\left[-q \mathbf{E}(t) \cdot v_{t}\right] \\
& =\frac{1}{c^{2}} \int_{t_{0}} \int_{\theta} \int_{\phi}\left\{\hat{x}\left[\bar{v} \sin \theta \cos \phi \cos \omega_{\mathrm{cr}}\left(t-t_{0}\right)+\bar{v} \cos \theta \sin \omega_{\mathrm{cr}}\left(t-t_{0}\right)\right]\right. \\
& \left.+\hat{y} \bar{v} \sin \theta \sin \phi+\hat{z}\left[\bar{v} \cos \theta \cos \omega_{\mathrm{cr}}\left(t-t_{0}\right)-\bar{v} \sin \theta \cos \phi \sin \omega_{\mathrm{cr}}\left(t-t_{0}\right)\right]\right\} \\
& \times q E \cos \omega t\left[\bar{v} \cos \theta \cos \omega_{\mathrm{cr}}\left(t-t_{0}\right)-\bar{v} \sin \theta \cos \phi \sin \omega_{c r}\left(t-t_{0}\right)\right] \\
& \times n_{\mathrm{e}} v_{\mathrm{r}} \mathrm{d} t_{0} \frac{\sin \theta \mathrm{d} \phi \mathrm{d} \theta}{4 \pi} \exp \left[-\frac{\bar{v}}{\lambda_{\mathrm{r}}}\left(t-t_{0}\right)\right] \\
& =n_{\mathrm{e}} \frac{\bar{v}^{2}}{3 c^{2}}[-q \mathbf{E}(t)]
\end{aligned}
$$


5) About $\sum \frac{m_{\mathrm{e}}}{\left(1-\frac{v\left(t_{0}\right)^{2}}{c^{2}}\right)^{1 / 2}} \frac{\partial \boldsymbol{v}\left(t, t_{0}\right)}{\partial t}$ in (3)

The above summation is a momentum which $n_{\mathrm{e}}$ electrons gain during unit time through the external fields and the collisions. Here, we assume roughly that a velocity distribution of $n_{\mathrm{e}}$ electrons at time $t$ is isotropic when it is viewed from the velocity point $\mathbf{u}(t)$, similarly in Figure 1 of Ref. [11]. Then, based on the analysis from (4) to (7), we have, as a momentum summation of $n_{\mathrm{e}}$ electrons at time $t$,

$$
\sum m_{\mathrm{e}} v\left(t, t_{0}\right)\left(1-\frac{v\left(t_{0}\right)^{2}}{c^{2}}\right)^{-\frac{1}{2}}=n_{\mathrm{e}} \frac{m_{\mathrm{e}}}{\gamma_{\mathrm{r}}}\left(1+\frac{\bar{v}^{2}}{3 c^{2}}\right) \mathbf{u}(t)
$$

Thus, we obtain the following momentum transport equation for relativistic electrons:

$$
\begin{aligned}
& n_{\mathrm{e}} \frac{m_{\mathrm{e}}}{\gamma_{\mathrm{r}}}\left(1+\frac{\bar{v}^{2}}{3 c^{2}}\right) \frac{\mathrm{d} \mathbf{u}(t)}{\mathrm{d} t}+n_{\mathrm{e}} \omega_{\mathrm{cr}} \tau \frac{\bar{v}^{2}}{c^{2}}\left[-\left(\beta_{2}+\frac{\beta_{2}^{\prime}}{\omega} \frac{\partial}{\partial t}\right)(-q \mathbf{E}(t) \times \hat{b})\right. \\
& \left.+\left(\beta_{1}+\frac{\beta_{1}^{\prime}}{\omega} \frac{\partial}{\partial t}\right) \hat{b} \times(-q \mathbf{E}(t) \times \hat{b})\right]+n_{\mathrm{e}} \frac{\bar{v}^{2}}{3 c^{2}}[-q \mathbf{E}(t)] \\
& =n_{\mathrm{e}}[-q \mathbf{E}(t)-q \mathbf{E}(t) \times \mathbf{B}]+\boldsymbol{P}_{\text {after }}-\boldsymbol{P}_{\text {before }}
\end{aligned}
$$

Here, with $\kappa=1 / 3$ and $\tau=1 / v_{\mathrm{r}}$,

$$
\begin{gathered}
\beta_{1}=\kappa \frac{-\omega_{\mathrm{cr}} \tau\left(\omega_{\mathrm{cr}}^{2} \tau^{2}-\omega^{2} \tau^{2}+1\right)}{D}, \beta_{1}^{\prime}=\kappa \frac{2 \omega_{\mathrm{cr}} \tau \omega \tau}{D}, \\
\beta_{2}=\kappa \frac{\omega_{\mathrm{cr}}^{2} \tau^{2}+\omega^{2} \tau^{2}+1}{D}, \beta_{2}^{\prime}=\kappa \frac{\omega \tau\left(\omega_{\mathrm{cr}}^{2} \tau^{2}-\omega^{2} \tau^{2}-1\right)}{D}, \\
\beta_{20}=\beta_{2\left(\omega_{\mathrm{cr}}=0\right)}=\frac{\kappa}{1+\omega^{2} \tau^{2}}, \beta_{20}^{\prime}=\beta_{20\left(\omega_{\mathrm{cr}}=0\right)}^{\prime}=\frac{-\kappa \omega \tau}{1+\omega^{2} \tau^{2}}, \\
D=\left(\omega_{\mathrm{cr}}^{2} \tau^{2}-\omega^{2} \tau^{2}+1\right)^{2}+4 \omega^{2} \tau^{2} .
\end{gathered}
$$

It is noted that (21) is the transport equation in the case where all electrons have the same velocity $\bar{v}$ (the mean thermal velocity).

\section{3. $v_{\mathrm{r}}, \varsigma_{\mathrm{r}}$ and Drift Velocities}

We regard, similarly in Equations (21)-(25) of Ref. [11], that a momentum transfer frequency of relativistic electrons scattered anisotropically in the two-body collisions through the Coulomb force is $v_{\mathrm{r}}\left(1-\varsigma_{\mathrm{r}}\right)$. Based on (A3) ${ }_{(\ell \rightarrow \infty)}$ in Appendix of Ref. [1] and the classical procedure, a relativistic collision cross section $\sigma_{\mathrm{r}}(x)$ in the electron-deuteron collisions through the Coulomb force is obtained as

$$
\sigma_{x}(x)=\frac{1}{4}\left(\frac{q^{2} \gamma_{\mathrm{r}}}{4 \pi \varepsilon_{0} m_{\mathrm{e}} \bar{v}^{2}}\right)^{2} \frac{1}{\sin ^{4} \frac{x}{2}}
$$

where, $x$ is a deflection angle of an electron with $\bar{v}$ and $\varepsilon_{0}$ is the dielectric 
constant of vacuum. Then, we obtain

$$
v_{\mathrm{r}}\left(1-\varsigma_{\mathrm{r}}\right)=n_{\mathrm{p}} \pi p_{\mathrm{up}-\mathrm{r}}^{2} \bar{v}\left[4\left(\frac{p_{\perp \mathrm{r}}}{p_{\mathrm{up}-\mathrm{r}}}\right)^{2} \ln \frac{p_{\mathrm{up}-\mathrm{r}}}{p_{\perp \mathrm{r}}}\right]
$$

Here, $p_{\text {up-r }}$ is an effective radius of the Coulomb force of a deuteron and $p_{\perp \mathrm{r}}=p_{\perp} \gamma_{\mathrm{r}}\left(p_{\perp}=q^{2} / 4 \pi \varepsilon_{0} m_{\mathrm{e}} \bar{v}^{2}\right)$ which is an impact parameter for $\pi / 2$-deflecttion in the relativistic electron-deuteron collisions. We presume that $p_{\mathrm{up}-\mathrm{r}}, v_{\mathrm{r}}$ and $\varsigma_{\mathrm{r}}$ are

$$
\left\{\begin{array} { l } 
{ p _ { \text { up } - \mathrm { r } } = p _ { \text { up } } \gamma _ { \mathrm { r } } } \\
{ v _ { \mathrm { r } } = n _ { \mathrm { p } } \pi p _ { \mathrm { up } - \mathrm { r } } ^ { 2 } \overline { v } = v \gamma _ { \mathrm { r } } ^ { 2 } } \\
{ 1 - \varsigma _ { \mathrm { r } } = 4 ( \frac { p _ { \perp \mathrm { r } } } { p _ { \text { up } - \mathrm { r } } } ) ^ { 2 } \operatorname { l n } \frac { p _ { \mathrm { up } - \mathrm { r } } } { p _ { \perp \mathrm { r } } } = 1 - \varsigma }
\end{array} \quad \left(p_{\mathrm{up}}\right.\right. \text { is given in (15) of Ref. [10]) }
$$

In (24), $v$ and $\varsigma$ are the quantities for the nonrelativistic case.

Now, when the external force fields are

$$
\mathbf{E}=-\hat{z} E, \mathbf{B}=\hat{y} B,
$$

a solution of the drift velocity $\mathbf{u}=\hat{x} u_{x}+\hat{z} u_{z}$ is given by

$$
\begin{aligned}
& \frac{u_{z}}{\frac{q \mathbf{E}}{m_{\mathrm{e}} v}}=\frac{1}{\gamma_{\mathrm{r}}} \cdot \frac{A_{1} A_{2}+A_{3} A_{4}}{A_{1}^{2}+A_{3}^{2}} \\
& \frac{u_{x}}{\frac{q \mathbf{E}}{m_{\mathrm{e}} v}}=\frac{1}{\gamma_{\mathrm{r}}} \cdot \frac{-A_{1} A_{4}+A_{2} A_{3}}{A_{1}^{2}+A_{3}^{2}}
\end{aligned}
$$

(note: $\left.q \mathbf{E} / m_{\mathrm{er}} v_{\mathrm{r}}=\left(q \mathbf{E} / m_{\mathrm{e}} v\right) / \gamma_{\mathrm{r}}\right)$

$$
\begin{gathered}
A_{1}=1-\varsigma_{\mathrm{r}}\left(1+\frac{\alpha}{3}\right)+\beta_{2} \varsigma_{\mathrm{r}}(1+\alpha) \\
A_{2}=1-\frac{\alpha}{3}-\beta_{2} \gamma_{\mathrm{r}}^{2}-\beta_{1} \omega_{\mathrm{cr}} \tau \alpha \\
A_{3}=\omega_{\mathrm{cr}} \tau+\beta_{1} \varsigma_{\mathrm{r}}(1+\alpha) \\
A_{4}=\beta_{2} \omega_{\mathrm{cr}} \tau \alpha-\beta_{1} \gamma_{\mathrm{r}}^{2} \\
\alpha=\bar{v}^{2} / c^{2}, \gamma_{\mathrm{r}}=(1-\alpha)^{1 / 2}
\end{gathered}
$$

The value of $\varsigma_{\mathrm{r}}(=\varsigma)$ can be regarded to be nearly 1.0 from (17) of Ref. [1].

We show in Figure 1 and Figure 2 variations of " $u_{z}$ in (26) and $u_{x}$ in (27)" with respect to $\omega_{\mathrm{c}} / v$ where $\omega_{\mathrm{c}}$ is the nonrelativistic cyclotron frequency.

When $\alpha \ll 1.0$ and $\varsigma_{\mathrm{r}} \simeq 1.0$,

$$
\begin{aligned}
& u_{z} /\left(q \mathbf{E} / m_{\mathrm{e}} v\right) \begin{cases}\simeq 2 / \gamma_{\mathrm{r}} & \left(\omega_{\mathrm{c}} / v=0\right) \\
\simeq \frac{1}{3\left(\omega_{\mathrm{c}}^{2} / v^{2}\right)} & \left(\omega_{\mathrm{c}} / v \rightarrow \infty\right)\end{cases} \\
& u_{\mathrm{x}} /\left(q \mathbf{E} / m_{\mathrm{e}} v\right) \begin{cases}=0 & \left(\omega_{\mathrm{c}} / v=0\right) \\
\simeq \frac{1}{\omega_{\mathrm{c}} / v} & \left(\omega_{\mathrm{c}} / v \rightarrow \infty\right)\end{cases}
\end{aligned}
$$




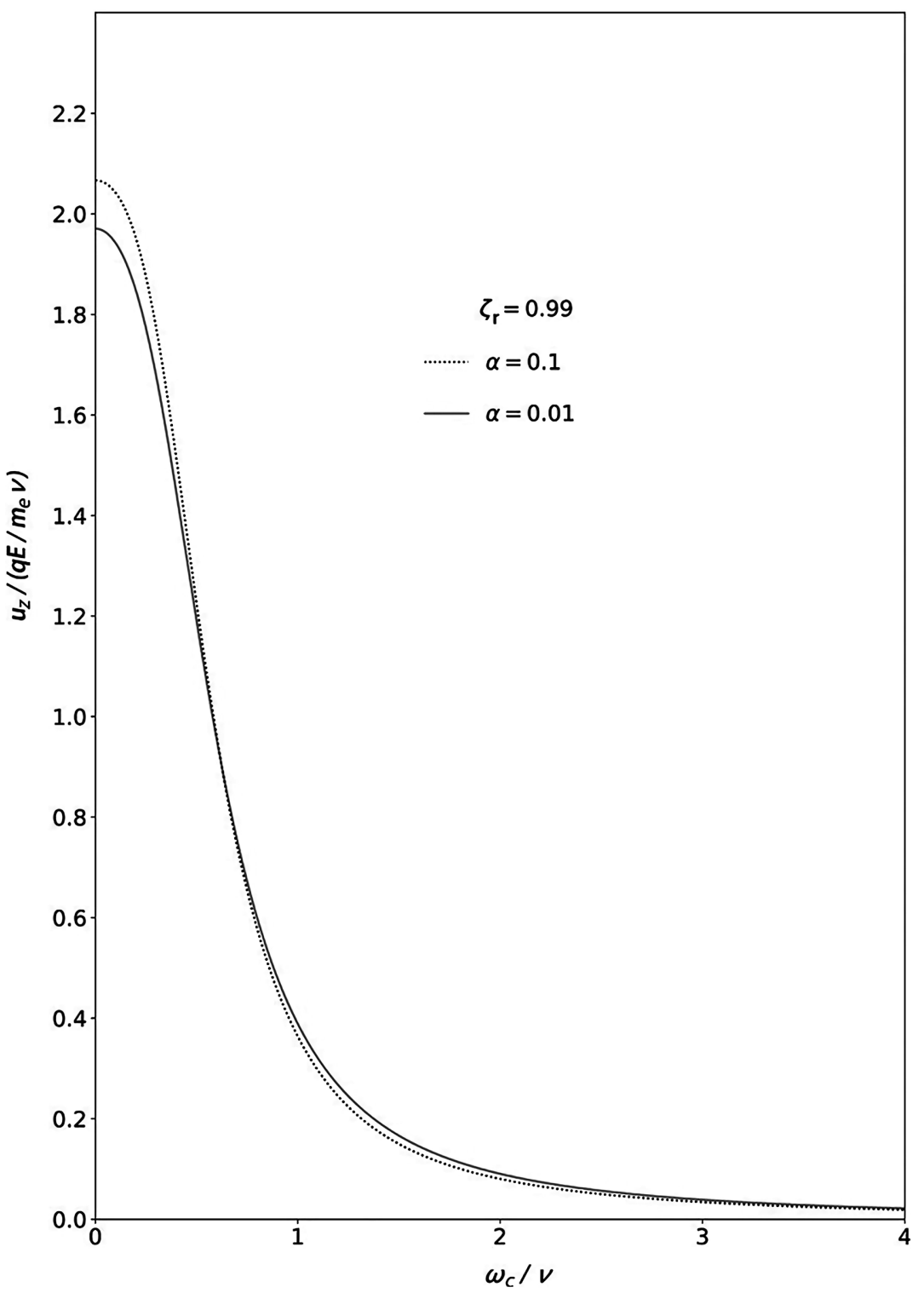

Figure 1. The drift velocity (26) of an electron. The quantities $v, m_{\mathrm{e}}, \omega_{\mathrm{c}}\left(=q B / m_{\mathrm{e}}\right)$ are the ones in the nonrelativistic case. $\alpha=\bar{v}^{2} / c^{2}$.

\section{For Efficiency Increase of a Thermionic Energy Converter}

It is presumed that a gas (Cs plasma) within general converters will be a weakly ionized plasma. If the gas is replaced with a fully ionized plasma instead of a weakly ionized one, an internal resistance between an emitter plate and a collector plate extremely decreases, and thermionic electrons can save their thermal energies which have been consumed for ionization of $\mathrm{Cs}$ atoms. We consider that this replacement of the internal medium will raise a generation-efficiency. Furthermore, if a force field to convey thermionic electrons from the emitter to the collector is given within the converter, the efficiency will rise more compared with the case where electrons cannot but go to the collector for themselves. Under such a consideration, we propose a means adding some equipments, shown 


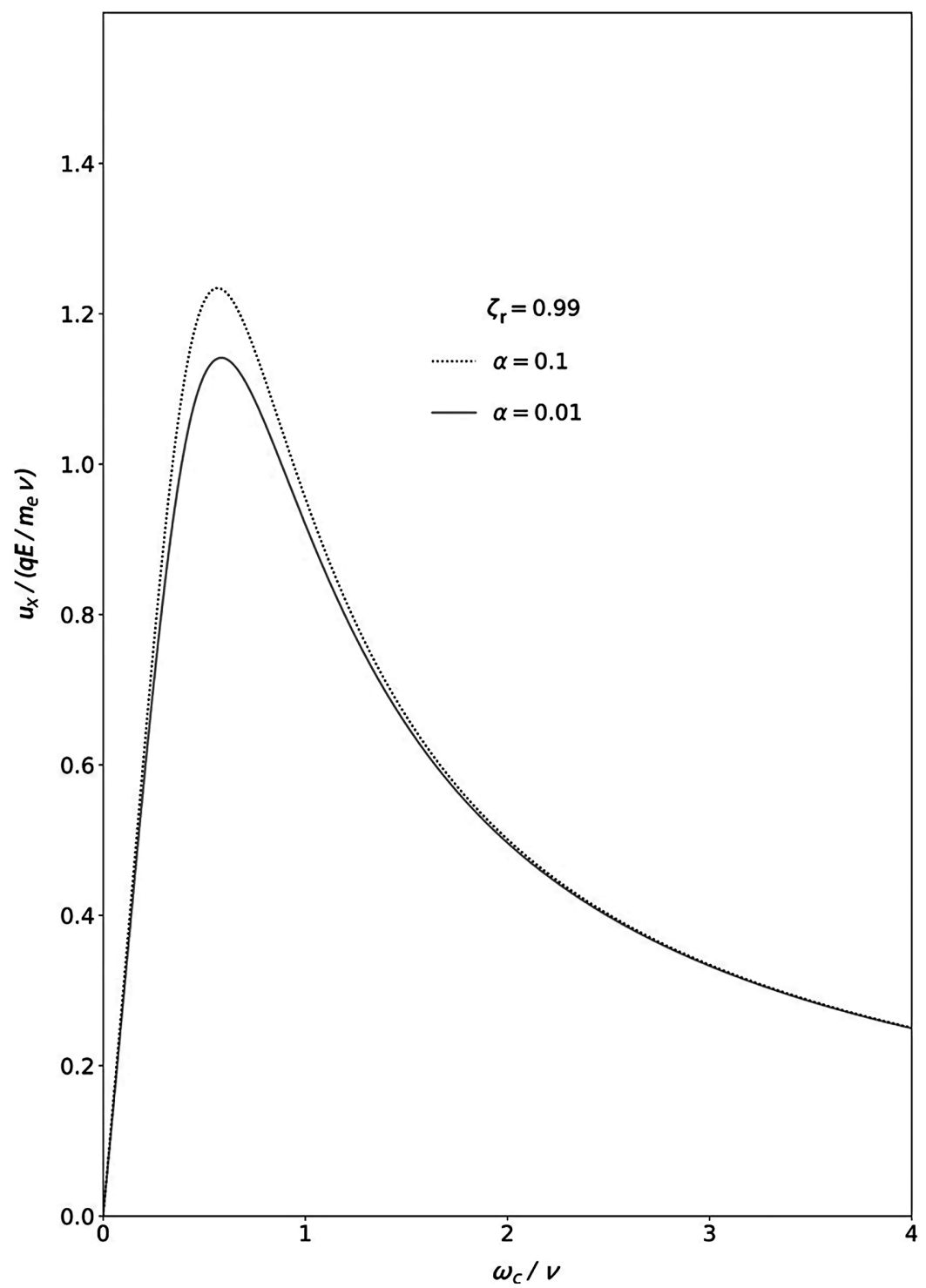

Figure 2. The drift velocity (27) of an electron. The quantities $v, m_{\mathrm{e}}, \omega_{\mathrm{c}}\left(=q B / m_{\mathrm{e}}\right)$ are the ones in the nonrelativistic case. $\alpha=\bar{v}^{2} / c^{2}$.

in Figure 3 and Figure 4, to the converter. The equipments are connected with Converter by Solenoid. In the inner space, Cs gas is enclosed. Discharge tube (shown in Figure 4) is installed as a partner of Converter. Fan makes Cs gas plasma circulate slowly within the closed space. By making a right-circularly polarized wave continue to heat electrons for long time, it is planned that the most part of the closed space is filled with a fully ionized plasma. Even if the electron temperature is not so high, we consider that it is possible to obtain an almost perfectly ionized plasma because the work-function of a Cs atom is very small. Now, let us classify the internal space of Converter into three parts (called space $1,2,3)$, as shown with dotted lines in Figure 3. We assume roughly that, in space 2, an electric field due to a space charge is negligible and also that, only in space 2, a magnetic field $\mathbf{B}$ and an external electric field $\mathbf{E}$ exist. The magnetic 


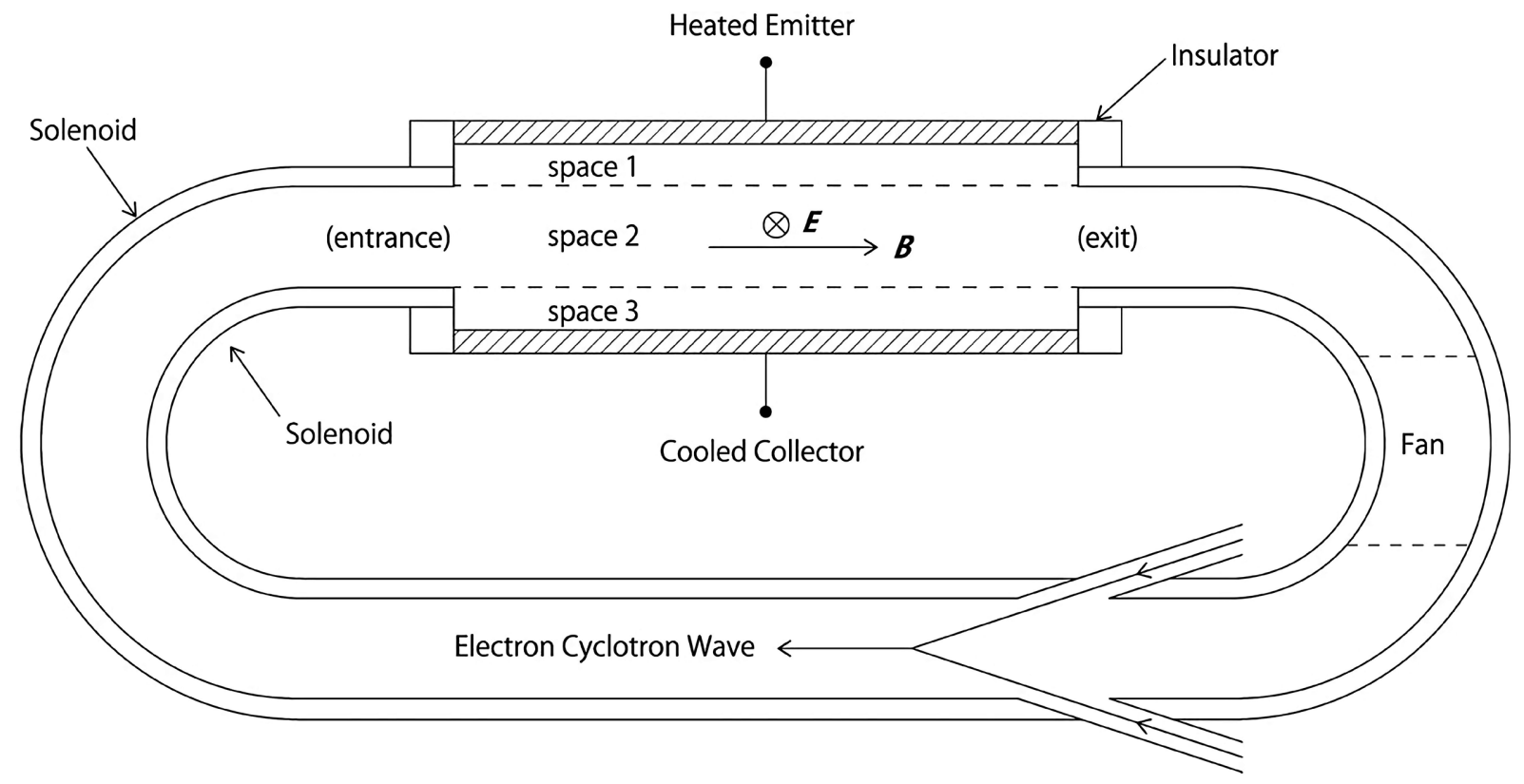

Figure 3. A fundamental structure of a thermionic energy converter which is connected with an Electric Wave Oscillator and a Fan by a rectangular Solenoid.

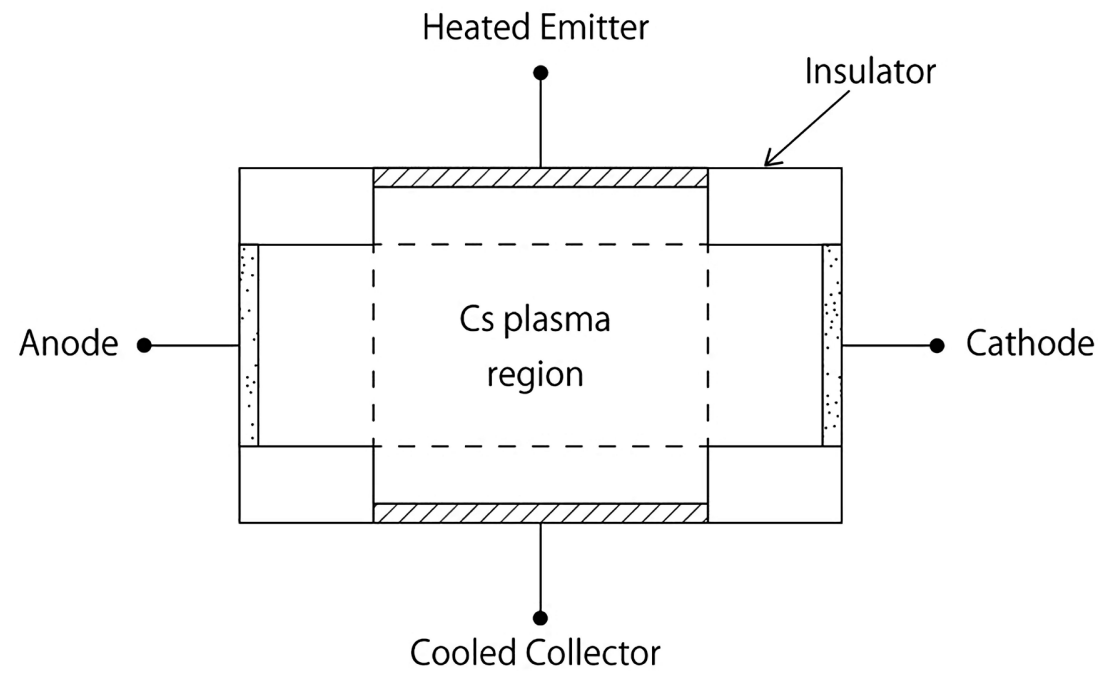

Figure 4. A side view of the converter with a Built-in Dischage Tube.

field B is supplied by Solenoid and the electric field E is the one in the middle part of the discharge plasma. These external forces convey electrons with the drift velocity $u_{z}$ (in Figure 1) in the direction of $-\mathrm{E}$ and with the drift velocity $u_{x}$ (in Figure 2) in the direction of $\mathbf{E} \times \mathbf{B}$. We set the value of $\omega_{\mathrm{c}} / v$ to a larger one than 4.0. Then, a loss in an external circuit between Anode and Cathode is sufficiently suppressed and many electrons will try entering from space 2 into space 3. In this situation, however, the following physical condition must be satisfied: "A total number of electrons which can enter within Collector per unit time is equal to a total number (denoted by $N_{\text {tot }}$ ) of electrons which jump out of Emitter per unit time." Electrons also combining with ions on Emitter must 
be counted for $N_{\text {tot }}$ (we suppose that neutral atoms produced near the surface of Emitter are soon ionized within space 1). Accordingly, if we design the converter so that electrons flowing into space 3 per unit time may become much more than $N_{\text {tot }}$, a negative potential barrier to suppress the flow of electrons ought to be produced near Collector surface, which is added to the old barrier. The larger the height of a total negative potential barrier becomes, the larger an output voltage becomes, because a potential of Collector lowers more and more as against a potential of Emitter. The convey of electrons by the force $\mathbf{E} \times \mathbf{B}$ makes it possible to lengthen the distance between Emitter and Collector. When the distance $\ell_{c}$ from the entrance to the exit in the converter of Figure 3 is too long, a distribution of $\mathbf{B}$ becomes vague. If it is necessary to lengthen the value of $\ell_{\mathrm{c}}$, then, we must connect some small-sized converters in series by solenoids.

\section{Conclusion}

In the field of the Coulomb force scattering, under the premise that the twobody collisions have much more influence than the many-body collisions on the drift movement of an electron, we have inquired into the transport equation of momentum for relativistic electrons. Also, proposing an idea of introducing a fully ionized plasma and an external magnetic field within the combination-apparatus of the converter and the discharge tube, we have discussed about a means to raise a generation-efficiency of a thermionic energy converter.

\section{Acknowledgements}

I express my sincere thanks for helpful discussions on this research and also for encouragement and help I have received for many years, to President K. Sawada of Soft Creator Company and Chief C. Adachi of Heian Light Technology Company.

\section{Conflicts of Interest}

The author declares no conflicts of interest regarding the publication of this paper.

\section{References}

[1] Nagata, M. (2020) Journal of Modern Physics, 11, 1751-1760. https://doi.org/10.4236/jmp.2020.1111108

[2] Rasor, N.S. (1982) Applied Atomic Collision Physics, 5, 169-200. https://doi.org/10.1016/B978-0-12-478805-3.50011-4

[3] Stakhanov, I.P. (1968) Soviet Physics-Technical Physics, 12, 1522.

[4] Miskolczy, G., Wang, C.C. and Lieb, D.P. (1981) Proceeding of 16 th Intersociety Energy Conversion Engineering Conference, 2, 1956.

[5] Huffman, F., Reagan, P., Miskolczy, G. and Merrill, O. (1982) Proceeding of 17 th Intersociety Energy Conversion Engineering Conference, 3, 1908.

[6] Huffman, F. (1983) Overview of Terrestrial Thermionics. 18th Intersociety Energy Conversion Engineering Conference, Orlando, 21 August 1983, p. 173. 
[7] Rasor, N.S. and Warner, C. (1964). Journal of Applied Physics, 35, 2589. https://doi.org/10.1063/1.1713806

[8] Norcross, D.W. and Stone, P.M. (1968) Journal of Quantitative Spectroscopy and Radiative Transfer, 8, 655-684. https://doi.org/10.1016/0022-4073(68)90181-7

[9] Cameron, G.E. and Reynolds, E.L. (1994) 29th Intersociety Energy Conversion Engineering Conference, 1, 389.

[10] Nagata, M. and Sawada, K. (2019) Journal of Modern Physics, 10, 145-156. https://doi.org/10.4236/jmp.2019.102011

[11] Nagata, M. (2011) The European Physical Journal D, 65, 429-440. https://doi.org/10.1140/epjd/e2011-10464-2 